\title{
THE SUPER GAGA PRINCIPLE AND FAMILIES OF SUPER RIEMANN SURFACES
}

\author{
PANKAJ TOPIWALA AND JEFFREY M. RABIN
}

(Communicated by Louis J. Ratliff, Jr.)

\begin{abstract}
We extend the GAGA principle, the Kodaira embedding theorem, and Chow's lemma to supergeometry and conclude that families of super Riemann surfaces are locally algebraic.
\end{abstract}

\section{O. INTRODUCTION}

A cornerstone of algebraic geometry is the principle that global analytic objects on a subvariety of projective space are actually algebraic, as detailed in Serre's fundamental paper [S2]. In particular, one deduces (Chow's lemma) that a complete analytic subvariety of projective space is algebraic, that is, the zero set of polynomial equations.

In recent years, an extension of ordinary geometry to a $\mathbf{Z}_{2}$-graded theory called supergeometry has been developed, largely for physical applications. It is natural to ask whether the above results continue to hold in this theory. In this paper, we provide a reading of [S2], in condensed form, modified as appropriate for supergeometry. The main consequence we are interested in is the Chow lemma, which has implications for the algebraicity of super Riemann surfaces (SRSs) [RaT]. See [LPW] for an independent proof.

Our formalization of supergeometry will be the standard sheaf-theoretic formulation as given for example by Manin [M2]. We review the main definitions.

Definitions. (1) A superscheme (resp. an analytic superspace) $\left(X, \mathscr{O}_{X}\right)$ consists of a topological space $X$ with a sheaf $\mathscr{O}_{X}=\mathscr{O}_{0} \oplus \mathscr{O}_{1}$ of supercommutative rings such that $\left(X, \mathscr{O}_{0}\right)$ is an ordinary scheme (resp. analytic space) and $\mathscr{O}_{1}$ is a coherent sheaf of $\mathscr{O}_{0}$-modules.

(2) A supermanifold of dimension $(p \mid q)$ is an analytic superspace $\left(X, \mathscr{O}_{X}\right)$ such that $\left(X, \mathscr{O}_{\text {red }}\right)$ is a manifold of dimension $p$, while $\mathscr{O}_{X}$ is locally isomor-

Received by the editors March 1, 1990 and, in revised form, June 13, 1990.

1980 Mathematics Subject Classification (1985 Revision). Primary 14E25, 14J10; Secondary $16 \mathrm{~A} 03,32 \mathrm{~J} 20$.

Key words and phrases. Super Riemann surfaces, projective superspace, Chow lemma, GAGA principle, Kodaira embedding theorem.

The first author was partially supported by NSF Grant DMS-861079. 
phic to $\Lambda(\mathscr{E})$ for a locally free sheaf $\mathscr{E}$ of rank $q . \mathscr{E}$ is then $\mathscr{I} / \mathscr{I}^{2}$, where $\mathscr{I}$ is the ideal of nilpotents in $\mathscr{O}_{X} . X$ is called split if $\mathscr{O}_{X}$ is globally $\Lambda(\mathscr{E})$. The tangent bundle $T_{X}$ is the (locally free rank $(p \mid q)$ ) sheaf of superderivations of $\mathscr{O}_{X}$.

(3) A morphism $\left(X, \mathscr{O}_{X}\right) \rightarrow\left(Y, \mathscr{O}_{Y}\right)$ in any of these categories of objects is a continuous map $f: X \rightarrow Y$ together with a morphism $g: \mathscr{O}_{Y} \rightarrow f_{*} \mathscr{O}_{X}$ of sheaves of supercommutative rings which is local in the sense that $g_{x}: \mathscr{O}_{Y, f(x)} \rightarrow$ $\mathscr{O}_{X, x}$ satisfies $g_{x}\left(m_{f(x)}\right) \subset m_{x}, m_{x}$ being the maximal ideal of the stalk at $x$. A morphism of supermanifolds is an embedding if the reduced morphism is an embedding of manifolds and $g$ is surjective.

(4) A line bundle on a supermanifold is a locally free rank 1 sheaf of $\mathscr{O}_{X^{-}}$ modules. (Thus it has both even and odd sections.)

Definition. Projective superspace $\mathbf{P}^{m \mid n}$ is the superscheme $\left(\mathbf{P}^{m}, \Lambda\left(\bigoplus_{1}^{n} \mathscr{O}(-1)\right)\right)$. $\mathbf{P}^{m \mid n}$ can be viewed as the space of lines $C^{1 \mid 0}$ in

$$
\begin{aligned}
\mathbf{C}^{m+1 \mid n} & =\operatorname{Spec} \mathbf{C}\left[x_{0}, \ldots, x_{m} ; \boldsymbol{\xi}_{1}, \ldots, \boldsymbol{\xi}_{n}\right] \\
& =\operatorname{Spec} \mathbf{C}\left[x_{0}, \ldots, x_{m}\right] \otimes \Lambda\left(\xi_{1}, \ldots, \boldsymbol{\xi}_{n}\right),
\end{aligned}
$$

an affine superscheme whose $\mathbf{Z}_{2}$ grading comes from $\operatorname{deg} x_{i}=0, \operatorname{deg} \xi_{i}=1$. $\mathbf{P}^{m \mid n}$ can also be regarded as a split supermanifold of dimension $(m \mid n)$ with a covering by the patches

$$
U_{i}=\left\{x_{i} \neq 0\right\}=\operatorname{Spec} \mathbf{C}\left[\frac{x_{0}}{x_{i}}, \ldots, \frac{x_{m}}{x_{i}} ; \frac{\xi_{1}}{x_{i}}, \ldots, \frac{\xi_{n}}{x_{i}}\right], \quad i=0, \ldots, m,
$$

with transition maps $x_{i} / x_{j}$ as usual.

Definition. An algebraic supervariety in $\mathbf{P}^{m \mid n}$ is a subsuperscheme $\left(X,\left.\left(\mathscr{O}_{\mathbf{P}^{m \mid n}} / I\right)\right|_{X}\right)$ with $I$ the ideal generated by finitely many homogeneous polynomials of definite $\mathbf{Z}_{2}$ parity.

$\mathbf{P}^{m \mid n}$ may be roughly viewed geometrically as the bundle $V=\bigoplus_{1}^{n} \mathscr{O}(1)$ on $\mathbf{P}^{m}$ (the sign change occurs because functions on a bundle live in the dual bundle). From ordinary GAGA, $V$ is an algebraic variety, and any global analytic subvariety of its holomorphic version $V^{h}$ (see [S2]) which is fiberwise algebraic must actually be algebraic. This is deduced, for example, by compactifying $V$ to $\mathbf{P}(V \oplus \mathscr{O})$, completing the subvariety and invoking GAGA there. Morally, this implies the super Chow lemma (and super GAGA generally), since an analytic subvariety of $\mathbf{P}^{m \mid n}$ may be viewed as a subvariety of $V$. (More accurately, its ideal sheaf is a subsheaf of $\Lambda\left(V^{*}\right)^{h}$.)

However, the difficulty in compactifying the "odd" dimensions in a supermanifold precludes this approach to applying GAGA. Nevertheless, GAGA does imply super GAGA in the following sense. For an analytic subsuperspace $\left(X, \mathscr{O}_{X}\right)$ of $\mathbf{P}^{m \mid n}$, with $\mathscr{O}_{X}=\mathscr{O}_{0} \oplus \mathscr{O}_{1}$, the ringed space $\left(X, \mathscr{O}_{0}\right)$ is an ordinary analytic space (with even nilpotents), and the extra structure then is simply a 
coherent $\mathscr{O}_{0}$-module $\mathscr{O}_{1}$, together with a multiplication map $\mathscr{O}_{1} \otimes_{\mathscr{O}_{0}} \mathscr{O}_{1} \rightarrow \mathscr{O}_{0}$. Now ordinary GAGA implies that the sheaf of rings $\mathscr{O}_{0}$, the $\mathscr{O}_{0}$-module $\mathscr{O}_{1}$ and the map $\mathscr{O}_{1} \otimes \mathscr{O}_{1} \rightarrow \mathscr{O}_{0}$ must all be algebraic, showing that an analytic subvariety of $\mathbf{P}^{m \mid n}$ must be "abstract-algebraic," that is, covered by affine patches having rational transition maps. Showing that it is actually a projective algebraic variety requires an additional argument that its ideal sheaf is generated by polynomials; this is the content of our super Chow theorem in $\S 1$.

To provide the proper setting for our super Chow theorem, we redo GAGA (quickly) in our context. Note that the arguments reduce as in [S2] to showing finite dimensionality of cohomology, and vanishing of higher cohomology for twisted sheaves, in the algebraic case. We leave this task, itself not conceptually difficult, to the ambitious reader; see [S1].

\section{Super GAGA}

$\mathbf{P}^{m}$ is both an algebraic and an analytic variety, where the topology is the Zariski or the complex, respectively. For $\mathbf{P}^{m \mid n}$, we have a similar dichotomy: the point set is $\mathbf{P}^{m}$, and for $U$ Zariski open, $\mathscr{O}_{\mathbf{P}^{m \mid n}}(U)=$ rational functions on $U$, whereas for $V$ complex open, $\mathscr{O}_{\mathbf{P}^{m \mid n}}^{h}(V)=$ holomorphic functions on $V$. In general, for $X \subset \mathbf{P}^{m \mid n}$ an algebraic subvariety, $\mathscr{F}$ an algebraic coherent sheaf, their holomorphic versions are denoted by $X^{h}, \mathscr{F}^{h}$ (e.g., $\left.\mathscr{F}^{h}=\mathscr{F} \otimes_{\mathscr{O}_{\mathbf{p}^{m \mid n}}} \mathscr{O}_{\mathbf{P}^{m \mid n}}^{h}\right)$. For details of these definitions, see Serre [S2]. Note that since the complex topology is finer, there is a continuous map $X^{h} \rightarrow X$, which is pointwise the identity.

We now prove:

Theorem 1. For all $q, H^{q}(X, \mathscr{F}) \tilde{\rightarrow} H^{q}\left(X^{h}, \mathscr{F}^{h}\right)$.

Theorem 2. $\operatorname{Hom}_{\mathscr{O}_{x}}(\mathscr{F}, \mathscr{G}) \stackrel{\sim}{\rightarrow} \operatorname{Hom}_{\mathscr{O}_{x}^{h}}\left(\mathscr{F}^{h}, \mathscr{G}^{h}\right)$.

Theorem 3. For $F$ an analytic coherent sheaf on $X^{h}$, there exists uniquely an algebraic coherent sheaf $\mathscr{F}$ on $X$ such that $\mathscr{F}^{h} \cong F$.

Proof of Theorem 1. Extend $\mathscr{F}$ by 0 to $\mathbf{P}^{m \mid n}$. Then $H^{q}(X, \mathscr{F}) \cong$ $H^{q}\left(\mathbf{P}^{m \mid n}, \mathscr{F}\right)$, and likewise in the analytic case. So it suffices to consider $X=\mathbf{P}^{m \mid n}$ from now on.

Lemma 1. True for $\mathscr{F}=\mathscr{O}$.

Proof. $\mathscr{O} \cong \Lambda\left(\bigoplus_{1}^{n} \mathscr{O}_{\mathbf{P}^{m}}(-1)\right)$. This reduces to a sum of $\mathscr{O}_{\mathbf{P}^{m}}(k)$, certain $k \leq 0$. Then Lemma 1 follows by direct calculation (Bott's formula), or by GAGA. Note that unlike the ordinary case, $H^{q}(\mathscr{O})$ may be nonzero for $q=m$ as well as $q=0$.

Lemma 2. True for $\mathscr{F}=\mathscr{O}(k)$. 
Proof. Induction on $k$ and $m$. Assume $k>0$. Let $E=$ even hyperplane $\cong \mathbf{P}^{m-1 \mid n}$. Consider the cohomology sequence for

$$
0 \rightarrow \mathscr{O}(-1) \rightarrow \mathscr{O} \rightarrow \mathscr{O}_{E} \rightarrow 0,
$$

yielding

$$
\begin{aligned}
& H^{q-1}\left(\mathscr{O}_{E}(k)\right) \rightarrow H^{q}(\mathscr{O}(k-1)) \rightarrow H^{q}(\mathscr{O}(k)) \rightarrow H^{q}\left(\mathscr{O}_{E}(k)\right) \rightarrow H^{q+1}(\mathscr{O}(k-1)) \rightarrow
\end{aligned}
$$

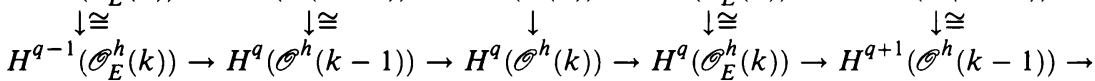

The induction hypothesis on $k, m$ gives the outer isomorphisms. The 5lemma gives an isomorphism in the middle. Note that $m=0$ is (still!) trivial, $k=0$ is above. The case $k<0$ is done by a downward induction.

End of Proof of Theorem 1. For general $\mathscr{F}$, there is a sequence (see [S1], and Remark at the end of $\S 1), 0 \rightarrow \mathscr{R} \rightarrow \mathscr{L} \rightarrow \mathscr{F} \rightarrow 0$ with $\mathscr{R}$ coherent, and $\mathscr{L}=\bigoplus_{1}^{r} \mathscr{O}(l)$, some $l, r$. Applying cohomology, we get a diagram

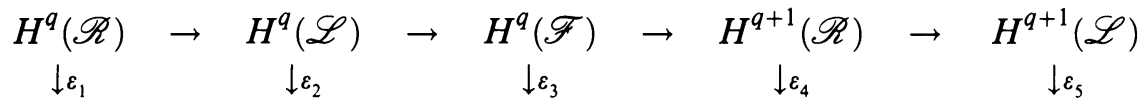

$$
\begin{aligned}
& H^{q}\left(\mathscr{R}^{h}\right) \rightarrow H^{q}\left(\mathscr{L}^{h}\right) \rightarrow H^{q}\left(\mathscr{F}^{h}\right) \rightarrow H^{q+1}\left(\mathscr{R}^{h}\right) \rightarrow H^{q+1}\left(\mathscr{L}^{h}\right) \text {. }
\end{aligned}
$$

Argue by induction on $q$, descending from $q=m+1$, and note that cohomology is 0 for $q>m$. Then, by the induction hypothesis, $\varepsilon_{4}$ and $\varepsilon_{5}$ are isomorphisms, and $\varepsilon_{2}$ is as well by Lemma 2. By the 5-lemma, $\varepsilon_{3}$ is onto. This being proved for arbitrary $\mathscr{F}$, in particular it is true for $\mathscr{F}=\mathscr{R}$, so that $\varepsilon_{1}$ is also onto.

By the 5-lemma again, we now get that $\varepsilon_{3}$ is actually an isomorphism.

Proof of Theorem 2. Let $\mathscr{A}=\underline{\operatorname{Hom}}_{\mathscr{O}_{X}}(\mathscr{F}, \mathscr{G})$ be the sheaf of germs of homomorphisms of $\mathscr{F}$ to $\mathscr{G}$, i.e., for $x \in X, f \in \mathscr{A}_{x}$ is a germ of a homomorphism $\mathscr{F} \rightarrow \mathscr{G}$ in a neighborhood of $x$. Let $\mathscr{B}=\underline{\operatorname{Hom}}_{\mathscr{O}_{x}^{h}}\left(\mathscr{F}^{h}, \mathscr{G}^{h}\right)$. Then we get a map $\mathscr{A}^{h} \stackrel{i}{\rightarrow} \mathscr{B}$ which we show is an isomorphism. Let $\mathscr{H}_{x} \equiv \mathscr{O}_{X, x}^{h}$, and abbreviate $\mathscr{O}_{X, x}$ as $\mathscr{O}_{X}$. Then

$$
\mathscr{A}_{x}=\operatorname{Hom}_{\mathscr{O}_{x}}\left(\mathscr{F}_{x}, \mathscr{G}_{x}\right), \quad \mathscr{A}_{x}^{h}=\operatorname{Hom}_{\mathscr{O}_{x}}\left(\mathscr{F}_{x}, \mathscr{G}_{x}\right) \otimes \mathscr{H}_{x},
$$

while

$$
\mathscr{B}_{x}=\operatorname{Hom}_{\mathscr{H}_{x}}\left(\mathscr{F}_{x} \otimes \mathscr{H}_{x}, \mathscr{G}_{x} \otimes \mathscr{H}_{x}\right),
$$

where all tensor products are over $\mathscr{O}_{x}$, and where we have used that $\mathscr{F}^{h} \otimes_{\mathscr{O}_{x}}$

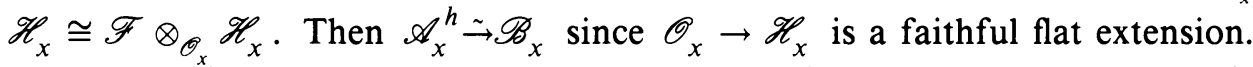
(For the ordinary case, see Serre [S2]. The extension to the super case here is elementary.) Finally, to prove Theorem 2, consider the maps

$$
H^{0}(X, \mathscr{A}) \stackrel{\varepsilon}{\rightarrow} H^{0}\left(X^{h}, \mathscr{A}^{h}\right) \stackrel{i}{\rightarrow} H^{0}\left(X^{h}, \mathscr{B}\right) .
$$

$i$ is an isomorphism by the above, and $\varepsilon$ is an isomorphism by Theorem 1 . But $H^{0}(X, \mathscr{A})=\operatorname{Hom}_{\mathscr{O}_{X}}(\mathscr{F}, \mathscr{G}), H^{0}\left(X^{h}, \mathscr{B}\right)=\operatorname{Hom}_{\mathscr{O}_{X}^{h}}\left(\mathscr{F}^{h}, \mathscr{G}^{h}\right)$. 
Proof of Theorem 3.

Uniqueness. By Theorem $2 \mathscr{F}^{h} \stackrel{\sim}{\rightarrow} \mathscr{G}^{h}$ implies that there exists a map $\mathscr{F} \stackrel{f}{\rightarrow} \mathscr{G}$ giving $\mathscr{F}^{h} \stackrel{\sim}{\rightarrow} \mathscr{G}^{h}$. Now faithful flatness of $\mathscr{O}_{X, x} \rightarrow \mathscr{O}_{X, x}^{h}$, for $x \in X$, implies $f$ is also an isomorphism.

Existence. Based on two lemmas. Use induction on $m$, with $m=0$ trivial.

Lemma 3. For $F$ an analytic coherent sheaf on $E \subset \mathbf{P}^{m \mid n}$ an even hyperplane, $H^{q}(E, F(k))=0$ for $q>0, k \gg 0$.

Proof. By the induction hypothesis, there is an algebraic coherent sheaf $\mathscr{F}$ such that $\mathscr{F}^{h}=F$. Therefore, $F(k)=\mathscr{F}^{h}(k)$, and by Theorem $1, H^{q}\left(E^{h}, F(k)\right) \cong$ $H^{q}(E, \mathscr{F}(k))$. Now the vanishing for $q>0, k \gg 0$ follows as in the ordinary algebraic case by Serre [S1]. See remark at the end of this section.

Lemma 4. $F(k)_{x}$ is generated by global sections for $k \gg 0$ and all $x$.

Proof. First note that if true for $k_{0}$, then also true for $k \geq k_{0}$, since we can multiply sections by $\left(x_{i} / x_{j}\right)^{k-k_{0}}$, where $\left\{x_{i}\right\}$ are homogeneous even coordinates. Also standard is the fact that if $F(k)_{x}$ is generated by global sections, so is $F(k)_{y}$, for $y$ near $x$. So if it is true that for all $x$ there exists $k$ such that $H^{0}(F(k))$ generates $F(k)_{x}$, then compactness implies we are done. To prove this, let $E$ be an even hyperplane, and consider the sequence

$$
\left(0 \rightarrow \mathscr{\mathscr { O }}^{h}(-1) \stackrel{\alpha}{\rightarrow} \mathscr{\mathscr { O }}^{h} \rightarrow \mathscr{O}_{E}^{h} \rightarrow 0\right) \otimes F .
$$

Then

$$
0 \rightarrow C \rightarrow F(-1) \stackrel{\alpha}{\rightarrow} F \rightarrow B \rightarrow 0,
$$

$C \equiv \operatorname{ker} \alpha, B \equiv \operatorname{coker} \alpha$. Twisting by $\mathscr{O}^{h}(k)$, we get

$$
0 \rightarrow C(k) \rightarrow F(k-1) \rightarrow F(k) \rightarrow B(k) \rightarrow 0 .
$$

Split this into two sequences:

$(*) \quad 0 \rightarrow C(k) \rightarrow F(k-1) \rightarrow P_{k} \rightarrow 0 \quad$ and $\quad 0 \rightarrow P_{k} \rightarrow F(k) \rightarrow B(k) \rightarrow 0$.

Now by definition, $\mathscr{O}^{h}(-1)=\mathscr{I}_{E}$, the ideal sheaf of $E$, so $\mathscr{I}_{E} B=0=\mathscr{I}_{E} C$, that is, $B, C$ live on $E$. By Lemma 3, there exists $k_{0}$ such that $H^{1}(B(k))=$ $0=H^{1}(C(k))$ for $k \geq k_{0}$. By $(*)$, applying cohomology, we conclude from vanishing that $h^{1}(F(k-1)) \geq h^{1}\left(P_{k}\right) \geq h^{1}(F(k))$, where $h^{1}$ means $\operatorname{dim} H^{1}$. Since $H^{1}(F(k-1))$ is finite-dimensional (again, Serre [S1] in the ordinary case, and Remark) we get that $h^{1}(F(k))$ stabilizes for large $k$. By the cohomology sequences for $(*)$, we conclude that

$$
H^{1}\left(P_{k}\right) \stackrel{\sim}{\rightarrow} H^{1}(F(k)) \Rightarrow H^{0}(F(k)) \rightarrow H^{0}(B(k)) \rightarrow 0,
$$

for large $k$. By the induction hypothesis of Theorem 3 again, $B \cong \mathscr{G}^{h}$, for some $\mathscr{G}$ algebraic coherent, and $\mathscr{G}(k)$ is generated by global sections implies $B(k)$ is generated by global sections. Let $M \equiv F(k)_{x},\left.N \equiv H^{0}(F(k))\right|_{x}$, 
$p \equiv I_{E_{x}}$. Then $B(k)_{x}=M \otimes \mathscr{O}_{X, x}^{h} / p, N \subset M$, and $M=N+p M$. If $m \supset p$ is a maximal ideal of $\mathscr{O}_{X, x}^{h}$, then also $M=N+m M$. By Nakayama's lemma, $N=M$.

End of Proof of Theorem 3. By Lemma 4,

$$
H^{0}(F(k)) \otimes \mathscr{O}^{h} \rightarrow F(k) \rightarrow 0 .
$$

If $r=\operatorname{dim} H^{0}(F(k))$, then

$$
0 \rightarrow R \rightarrow L_{0} \rightarrow F \rightarrow 0,
$$

where $R=$ kernel, $L_{0}=\bigoplus_{1}^{r} \mathscr{O}^{h}(l)$, some $l$ (in fact $l=-k$ ). Conclude that there is a sequence

$$
L_{1} \stackrel{g}{\rightarrow} L_{0} \rightarrow F \rightarrow 0
$$

for some $L_{1}=\bigoplus_{1}^{t} \mathscr{O}^{h}(s)$, some $s, t$. The algebraic versions of the sheaves $L_{0}$ and $L_{1}$ certainly exist; they are simply $\mathscr{L}_{0}=\bigoplus_{1}^{r} \mathscr{O}(-k)$ and $\mathscr{L}_{1}=\bigoplus_{1}^{t} \mathscr{O}(s)$. By Theorem 2, there exists an algebraic map $f$ such that $f^{h}=g$. Let $\mathscr{F}=$ coker $f$, so that

$$
\mathscr{L}_{1} \stackrel{f}{\rightarrow} \mathscr{L}_{0} \rightarrow \mathscr{F} \rightarrow 0 .
$$

Then

$$
L_{1} \stackrel{g}{\rightarrow} L_{0} \rightarrow \mathscr{F}^{h} \rightarrow 0
$$

is also exact, by flatness. Therefore $\mathscr{F}^{h}=F$.

Theorem (super Chow). If $X \subset \mathbf{P}^{m \mid n}$ is an analytic subvariety, then $X$ is algebraic.

Proof. The ideal sheaf $I_{X}$ is a coherent analytic sheaf, as is $\mathscr{O}_{X}=\mathscr{O} / I_{X}$. So there exists an algebraic sheaf $\mathscr{F}$ such that $\mathscr{F}^{h}=\mathscr{O}_{X}$. Now $\operatorname{supp} \mathscr{F}^{h}=$ $\operatorname{supp} \mathscr{F}=X$, showing $X$ is algebraic, i.e. Zariski closed.

Of course, we need to know if $X$ is given by a finite number of polynomials. That goes as follows.

For a sheaf $\mathscr{F}$ on $\mathbf{P}^{m \mid n}$, let $\Gamma_{*}(\mathscr{F}) \equiv \Gamma\left(\bigoplus_{k \in \mathbf{Z}} \mathscr{F}(k)\right), \mathscr{F}(k) \equiv \mathscr{F} \otimes_{\mathscr{O}} \mathscr{O}(k)$.

Lemma 5. $\Gamma_{*}\left(\mathscr{O}_{\mathbf{p}^{m \mid n}}\right)=C\left[x_{0}, \ldots, x_{m} ; \xi_{1}, \ldots, \xi_{n}\right]$.

Proof. Compute.

$$
\begin{aligned}
\Gamma\left(\bigoplus_{k \in \mathbf{Z}} \mathscr{O}(k)\right) & =\Gamma\left(\bigoplus_{k=0}^{\infty} \mathscr{O}(k)\right) \\
& =\bigoplus_{k=0}^{\infty} C\left\langle x_{0}^{k_{0}} \cdots x_{m}^{k_{m}} \xi_{1}^{l_{1}} \cdots \xi_{n}^{l_{n}}\right| k_{0}+\cdots+k_{m}+l_{1}+\cdots+l_{n}=k, \\
& =C\left[x_{0}, \ldots, x_{m} ; \xi_{1}, \ldots, \xi_{n}\right] .
\end{aligned}
$$


For a module $M$ over $A \equiv C\left[x_{0}, \ldots, x_{m} ; \xi_{1}, \ldots, \xi_{n}\right]$, let $\widetilde{M}$ be the $\mathscr{O}_{\mathbf{P}^{m \mid n}}$ sheaf over $\mathbf{P}^{m \mid n}$ such that $\widetilde{M}\left(U_{i}\right)=M_{\left(x_{i}\right)}$, the localization of $M$ at the multiplicative set generated by $x_{i}$, where $U_{i}=\left\{x_{i} \neq 0\right\}$.

Lemma 6. Let $\mathscr{F}$ be a coherent sheaf on $\mathbf{P}^{m \mid n}$. Then $\widetilde{\Gamma_{*}(\mathscr{F})} \stackrel{\sim}{\rightarrow} \mathscr{F}$.

Proof. $\mathbf{P}^{m \mid n}$ is covered by the $U_{i}=\left\{x_{i} \neq 0\right\}$. Define a map $\widetilde{\Gamma_{*}(\mathscr{F})} \rightarrow \mathscr{F}$ over $U_{i}$ as follows. An element of $\left.\Gamma_{*}^{(\mathscr{F}}\right)\left(U_{i}\right)=\Gamma_{*}(\mathscr{F})_{\left(x_{i}\right)}$ is of the form $f / x_{i}^{d}$, some $d \geq 0$, and $f \in \Gamma(\mathscr{F}(d))$. Send $f / x_{i}^{d} \rightarrow f \cdot x_{i}^{-d}$, defined in $\mathscr{F}\left(U_{i}\right)$ since $x_{i}^{-d}$ is holomorphic on $U_{i}$. This map is preserved under coordinate changes, and is clearly one-to-one. Surjectivity is provided by the fact that since $\mathscr{F}$ is coherent, $\mathscr{F}(d)$ is generated by its global sections, for some $d$ (see remark below).

Proposition. If $X \subset \mathbf{P}^{m \mid n}$ is a closed subscheme, then $X$ is the zero set of finitely many homogeneous polynomials in $A$. (This generalizes the result in [LPW], where $X$ is assumed smooth.)

Proof. $0 \rightarrow I_{X} \rightarrow \mathscr{O}_{\mathbf{P}^{m \mid n}}$, so $0 \rightarrow \Gamma_{*}\left(I_{X}\right) \rightarrow \Gamma_{*}\left(\mathscr{O}_{\mathbf{p}^{m \mid n}}\right)$ as is easily seen. Therefore, $\Gamma_{*}\left(I_{X}\right)$ is a submodule of $\Gamma_{*}^{*}\left(\mathscr{O}_{\mathbf{P}^{m \mid n}}\right)=A$, i.e. it is a homogeneous ideal. Since $\Gamma_{*}\left(I_{X}\right)=I_{X}$, its zero set is $X$. Now finiteness follows from the easy fact that $A$ is a noetherian ring.

Remark. Essentially, it remains to verify as in Serre [S1] that for an algebraic coherent sheaf $\mathscr{F}$ on $\mathbf{P}^{m \mid n}, H^{q}\left(\mathbf{P}^{m \mid n}, \mathscr{F}\right)$ is a finite-dimensional $C$-vector space, and vanishes for $\mathscr{F}(k), k \gg 0$, and $q>0$. These two facts then imply that $\mathscr{F}(k)$ is generated by its global sections, i.e.

$$
H^{0}(\mathscr{F}(k)) \otimes \mathscr{O}_{\mathbf{P}^{m \mid n}} \rightarrow \mathscr{F}(k) \rightarrow 0 .
$$

If $\operatorname{dim} H^{0}(\mathscr{F}(k))=r$, then we get

$$
0 \rightarrow \mathscr{R}^{\prime} \rightarrow \bigoplus_{1}^{r} \mathscr{O} \rightarrow \mathscr{F}(k) \rightarrow 0,
$$

or

$$
0 \rightarrow \mathscr{R} \rightarrow \bigoplus_{1}^{r} \mathscr{O}(-k) \rightarrow \mathscr{F} \rightarrow 0 .
$$

The existence of such a sequence was also used frequently above.

There appears to be little difficulty in extending this to the super case, save for much background material and notation. Essentially, since the $\xi$ 's are nilpotent, all sections of sheaves are polynomial in the $\xi_{i}$ anyway. When restricting to the algebraic category, we are restricting expressions in the even $x_{i}$ to be polynomial (or rational). The finite dimensionality and vanishing for twisted sheaves is then elementary. 


\section{FAMILIES OF SRSS}

In [RaT], we prove that SRSs are embeddable in projective superspace as algebraic subvarieties. (There we used the SRS structure explicitly.) Here we sketch a more general argument in sheaf-theoretic language [M1, M2].

Definition. A SRS $M$ is a (1|1)-dimensional supermanifold such that $M_{\text {red }}$ is an ordinary Riemann surface, and such that the tangent bundle $T_{M}$ has a rank (0|1) subbundle $\mathscr{D}$ with

$$
\mathscr{D}^{\otimes 2} \stackrel{\left[\mathrm{H}_{+}\right.}{\sim} T_{M} / \mathscr{D}
$$

More generally, a family of SRSs $X \stackrel{\pi}{\rightarrow} S$ over a supermanifold $S$ is a proper submersion of relative dimension (1|1) with a subbundle of $T_{X / S}$ as above. See [D, LRo] for these definitions and further elaboration.

First, we present a lemma which exploits the close analogy between supergeometry and "formal" deformation theory, that is, deformations over nilpotent parameters.

Lemma (super Kodaira). If $\left(X, \mathscr{O}_{X}\right)$ is an analytic supermanifold, $X$ compact, and $L$ is a line bundle on $X$ such that $L_{\mathrm{red}}$ is ample on $X_{\mathrm{red}}$, then $L$ is ample on $X$.

Note that this does not assert that every ample line bundle on $X_{\text {red }}$ is $L_{\text {red }}$ for some line bundle $L$ on $X$. The super Grassmannians in Manin's famous example fail to be projective for just this reason [M2].

Proof. Let $\mathscr{I}=$ ideal sheaf of nilpotents, so that

$$
0 \rightarrow \mathscr{I} \rightarrow \mathscr{O} \rightarrow \mathscr{O}_{\text {red }} \rightarrow 0 \text {. }
$$

We must show that for $\nu \gg 0$, sections of $L^{\nu}$ embed $X$ in $\mathbf{P}^{m \mid n}$, some $m, n$. This amounts to separating points and tangent vectors, which can be checked cohomologically [GHar]. For example, consider

$$
\left(0 \rightarrow I_{x^{2}} \rightarrow \mathscr{O} \rightarrow \mathscr{O} / I_{x^{2}} \rightarrow 0\right) \otimes \mathscr{I}^{k} / \mathscr{J}^{k+1} \otimes L^{\nu}
$$

where $I_{x^{2}}=$ ideal of functions vanishing to second order at $x \in X$. Now

$$
I_{x^{2}} \otimes \mathscr{I}^{k} / \mathscr{I}^{k+1} \otimes L^{\nu} \cong I_{x^{2}} \otimes \mathscr{I}^{k} / \mathscr{I}^{k+1} \otimes L_{\mathrm{red}}^{\nu}
$$

can be assumed to have $H^{1}()=0$, since $L_{\text {red }}$ is ample and $I_{x^{2}} \otimes \mathscr{J}^{k} / \mathscr{J}^{k+1}$ is $\mathscr{O}_{\text {red }}$-coherent. Since $\mathscr{J}^{k}=0$ for $k$ large, we get that $H^{1}\left(I_{x^{2}} \otimes L^{\nu}\right)=0$ for $\nu$ large, using

$$
\left(0 \rightarrow \mathscr{I}^{k} / \mathscr{I}^{k+1} \rightarrow \mathscr{O} / \mathscr{I}^{k+1} \rightarrow \mathscr{O} / \mathscr{I}^{k} \rightarrow 0\right) \otimes I_{x^{2}} \otimes L^{\nu},
$$

and inducting upwards starting at $k=1$.

This gives

$$
H^{0}\left(L^{\nu}\right) \rightarrow H^{0}\left(\mathscr{O} / I_{x^{2}} \otimes L^{\nu}\right) \rightarrow 0
$$


that is, $H^{0}\left(L^{\nu}\right)$ separates tangent vectors at $x$. Furthermore, by "semicontinuity," we get that nearby points and tangent vectors are also separated. While this was for a fixed point $x$, by compactness of $X$ we conclude that tangent vectors at all points are separated, for $\nu \gg 0$. By compactness of the complement of a neighborhood of the diagonal in $X \times X$, we conclude that any two points are separated for $\nu \gg 0$ (it is necessary to consider $I_{x, y} \equiv$ sheaf of functions vanishing at $x$ and $y$, to get the remaining separations).

Remark. For $X \stackrel{\pi}{\rightarrow} S$ an algebraic family, that is, $X, S$ are superschemes, and $\pi$ is a proper flat algebraic map, we get a similar conclusion in relative form, since in any case $X, S$ are quasicompact in the Zariski topology (we do not need $S$ to be "complete" here). For terminology, see [Hart].

Now for $M_{\text {red }}$ a compact Riemann surface of genus $g \geq 2$, it is well known that $T^{*} M_{\text {red }}$ is an ample line bundle. On a SRS $M$, or in a family $X \stackrel{\pi}{\rightarrow} S$, we conclude that $L=\mathscr{D}^{-2}$, with $L_{\text {red }}=T^{*} M_{\text {red }}$, is relatively ample. More generally, for $M$ a $(1 \mid n)$ supermanifold, we can construct an $L$ with $L_{\text {red }}=T^{*} M_{\text {red }}$ by taking the divisor of $L$ to consist of codimension $(1 \mid 0)$ hypersurfaces in $M$ whose reduction modulo nilpotents gives a divisor $D$ of $T^{*} M_{\text {red }}$. For example, choose an atlas $\left\{U_{i}\right\}$ on $M_{\text {red }}$ such that each point of $D$ lies in exactly one chart and define the hypersurfaces by the same local equations $f_{i}\left(z_{i}\right)=0$ which define the points of $D$, so that the transition map of $L$ on $U_{i} \cap U_{j}$ is $f_{i} / f_{j}$. Thus an SRS structure on $M$ is not required.

If the total space of a family $\left(X, \mathscr{O}_{X}\right) \rightarrow\left(Y, \mathscr{O}_{Y}\right)$ of analytic supermanifolds (e.g., SRSs) satisfies the hypotheses of our super Kodaira lemma, we get an embedding of this total space in some $\mathbf{P}^{m \mid n}$ which, by super Chow, realizes it as an algebraic supervariety. More frequently, however, the total space is noncompact and one wants a family of embeddings of the fibers, that is, a suitable map $\left(X, \mathscr{O}_{X}\right) \rightarrow \mathbf{P}^{m \mid n} \times\left(Y, \mathscr{O}_{Y}\right)$ which is "fiberwise algebraic." This can be done by an extension of our methods, but since the argument does not differ significantly from that of [LPW], we merely quote a version of their result:

Theorem (Le Brun-Poon-Wells). Let $\left(X, \mathscr{O}_{X}\right) \rightarrow\left(Y, \mathscr{O}_{Y}\right)$ be a proper family of supermanifolds carrying a line bundle with relatively ample reduction. Then any point $y \in Y$ has a neighborhood $U$ such that $\left.X\right|_{U}$ embeds in $\mathbf{P}^{m \mid n} \times\left(U, \mathscr{O}_{Y}(U)\right)$ respecting the projections to $U$ and with the ideal of $\left.X\right|_{U}$ generated by finitely many homogeneous polynomials in the coordinates of $\mathbf{P}^{m \mid n}$ with coefficients from $\mathscr{O}_{Y}(U)$.

In particular, families of SRSs are at least locally algebraic. It would be desirable to show that the moduli space of SRSs [D, LRo] is actually an algebraic superscheme, and to study it by algebraic methods. An important open problem here, with no analog in the usual theory of algebraic curves, is to characterize the moduli space of SRSs as a subscheme of the larger moduli space of (1|1)dimensional supercurves. 


\section{ACKNOWLEDGMENT}

Pankaj Topiwala thanks P. Deligne and M. Rothstein for useful conversations.

\section{REFERENCES}

[D] P. Deligne, Letter to Manin, Princeton, October 1987.

[GHar] P. Griffiths and J. Harris, Principles of algebraic geometry, Wiley, New York, 1978.

[Hart] R. Hartshorne, Algebraic geometry, Springer-Verlag, New York, 1977.

[LPW] C. LeBrun, Y.-S. Poon, and R. O. Wells, Projective embeddings of complex supermanifolds, Commun. Math. Phys. 126 (1990), 433-452.

[LRo] C. LeBrun and M. Rothstein, Moduli of super Riemann surfaces, Commun. Math. Phys. 117 (1988), 159-176.

[M1] Yu. Manin, New dimensions in geometry, Russian Math. Surv. 39:6 (1984), 51-83.

[M2] _ Gauge field theory and complex geometry, Springer-Verlag, New York, 1988.

[RaT] J. Rabin and P. Topiwala, Super Riemann surfaces are algebraic curves, Univ. California, San Diego, preprint, 1988.

[S1] J.-P. Serre, Faisceaux algébriques cohérents, Ann. of Math. 61 (1955), 197-278.

[S2] $\frac{1}{1-42}$, Géometrie algébrique et géometrie analytique, Ann. Inst. Fourier (Grenoble) 6 (1956),

Mitre Corporation, Mail E025, Burlington Road, Bedford, Massachusetts 01730

Department of Mathematics, University of California, San Diego, la Jolla, CaliforNIA 92093-0012 